\title{
Fundamental Acoustical Finger Force Calculation for Out-of-Plane Ultrasonic Vibration and its Correlation with Friction Reduction
}

\author{
Anis Kaci $^{1}$ Angelica Torres ${ }^{1}$, Frederic Giraud ${ }^{1}$, Christophe Giraud-Audine ${ }^{2}$ \\ Michel Amberg ${ }^{1}$ and Betty Lemaire-Semail ${ }^{1}$
}

\begin{abstract}
This article presents a method for estimating the parameters of a biomechanical impedance approximation model for a finger interacting with a rigid beam vibrating outof-plane at ultrasonic frequencies, and illustrates correlation results of these parameter magnitudes with vibration amplitude friction modulation detection threshold.
\end{abstract}

\section{INTRODUCTION}

Ultrasonic out-of-plane vibration is one of the leading technologies to create friction modulation in haptic devices. By modulating the friction forces between a vibrating plate and a user's finger as a function of its position, illusions of out of plane shapes and textural patterns can be created [1], [2]. The intermittent contact between the finger pad and the plate, that occurs during out-of plane vibrations at amplitudes as low as few micrometers, is at the origin of the friction reduction [3], [4]. It has been observed that the finger biomechanic help to predict the behavior of friction forces during tactile surface exploration. In [3], it is shown that soft stratum-corneum - with a low Young modulus - have lower friction reduction, while [5] has established that the damping of the stratum corneum at ultrasonic frequencies greatly affects the subject's susceptibility to ultrasonic friction modulation.

Therefore, it is conceivable that certain finger mechanical properties are correlated to a measure of the textural perception. This is further supported by [6], which has shown that the perceptual intensity of the tactile stimuli is a function of the friction contrast. This has been confirmed by [7], who has modeled this intensity by introducing also the change rate of the tangential force. Difference between falling and rising friction is observed, and is due to the viscoelastic properties of the fingertip.

Therefore, to obtain a same perceptual intensity of a tactile stimuli, the vibration amplitude should be adapted according to the scanning speed and pressing force, which can be measured with sensors, but also to the biomechanical properties of the finger pad, which change from one user to the other. In [8], the measured acoustical impedance is correlated to the perception of simulated keyclick, since this measure is directly correlated to the biomechanical properties of the finger pad. The authors propose to use this

\footnotetext{
*This work has been carried out within the framework of the Mint Project of IRCICA (Service and Research unit from CNRS)

${ }^{1,2}$ The authors are with Univ. Lille, Arts et Metiers ParisTech, Centrale Lille, HEI, EA 2697 - L2EP - Laboratoire d'Electrotechnique et d'Electronique de Puissance, F59000 Lille, France ${ }^{1}$ name.surnameduniv-lille.fr ${ }^{2}$ christophe.giraud-audine@ensam.eu
}

measurement as a monitoring tool to produce a calibrated stimulation to each user.

This paper explores the application of the measurement of the acoustic forces in the case of friction reduction tactile displays. For that purpose, we first present the principle of an online measurement of the forces. We the present an experimental study which aim is to correlate the force measurement to the friction reduction. the results of this study are then presented in the final section.

\section{PRINCIPLE OF THE FUNDAMENTAL ACOUSTICAL FORCE MEASUREMENT}

\section{A. Definition of the acoustical finger force}

We consider a vibrating plate which is actuated by piezoelectric cells supplied by a voltage $v(t)$ around a pulsation denoted $\omega$, and which vibration amplitude is named $W$. The voltage $v$ creates an internal force $f_{p}$ such that $f_{p}=N v$, which is used to compensate the internal friction force that exists due to the plate's particles motion, a phenomenon called internal damping. When a finger touches the vibrating plate, contact forces appear that can create additional damping and stiffness into the system formed by the plate and the finger. As a result, the voltage $v$ needs to be adapted, so that $f_{p}$ can compensate the effect of the contact forces on the vibration, and maintain $W$ constant [9]. Therefore, the voltage difference between the no-load condition and the contact condition is related to the contact forces, and thus to the biomechanical properties of the fingertip.

Without lack of generality, we consider the model of a vibrating plate which is operated exactly at its resonance frequency, leading to the equation 1 :

$$
D \dot{w}=f_{p}-f_{r}
$$

with $D$ the internal damping of the plate around a resonance pulsation, and $f_{r}$, the acoustical force. In general, $f_{r}$ derives from the contact pressure $p(x, y, t)$ which depend on time $t$ and position $\{x, y\}$. The theory of vibrating plate helps to write the relationship between $f_{r}$ and $p$, by introducing $\Psi(x, y)$, the modal shape of the plate when vibrating:

$$
f_{r}=\int_{S} p(x, y, t) \Psi(x, y) \mathrm{d} x \mathrm{~d} y
$$

where $S$ is the contact area.

It is then possible to observe $f_{r}$ by inverting the relation 1 , leading to:

$$
\tilde{f}_{r}=N v-D \dot{w}
$$


where $\tilde{f}_{r}$ represents the observation of $f_{r}$. It should be emphasized here that the acoustical finger force is not the contact force, but it derives from it.

However, the variables $w(t)$ and $v(t)$ are high frequency signals, and solving the equation 3 in the time domain can be achieved at the expense of high sampling rate, and off-line calculation. Therefore, we transform the observation into the rotating reference frame.

\section{B. Measurement in the rotating reference frame}

Assuming that $v$ is a sinusoidal function of time, one can use phasors to model it, hence:

$$
\underline{v}(t)=\left(V_{d}+j V_{q}\right) e^{j \omega t}
$$

where $V_{d}$ and $V_{q}$ represent the coordinates of $\underline{v}$ into a the rotating reference frame, and $\underline{v}$ is the phasor of $v$ such that $v(t)$ is the imaginary part of $\underline{v}$. This representation is illustrated figure 3.

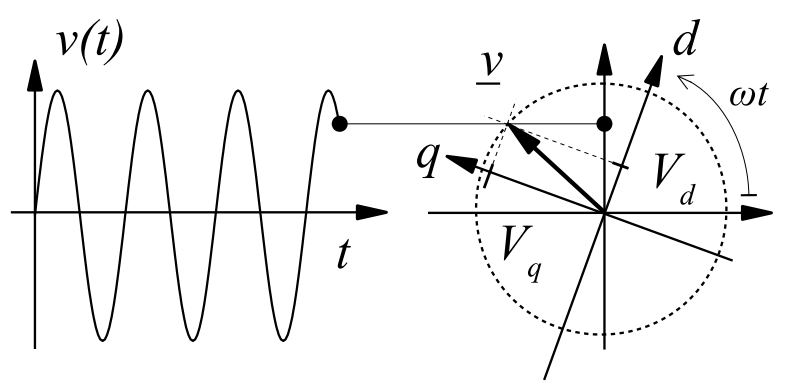

Fig. 1. Principle of the rotating reference frame $(d, q)$.

In the same way, we define $W_{d}, W_{q}, F_{r d}$ and $F_{r q}$. How to impose $V_{d}$ and $V_{q}$ and to control $W_{d}$ and $W_{q}$ is detailed in [10].

In the remaining of this paper, the plate is controlled in such a way that $W_{q}=0$, leading to $\underline{\dot{w}}=j \omega W_{d} e^{j \omega t}$; the equation 3 leads then to two new equations, after projecting on axis $d$ and $q$ :

$$
\tilde{F}_{r d}=N V_{d} \quad, \quad \tilde{F}_{r q}=N V_{q}-D \omega W_{d}
$$

\section{Theoretical examples}

For the purpose of illustration, we present three examples of acoustical finger force observation. In the first example, we consider no contact conditions, and the probing finger is absent, leading to $f_{r}=0$. Then, the equation 5 yields:

$$
V_{d}=0 \quad, \quad V_{q}=\frac{N}{D} \omega W_{d}
$$

Hence, at no load, $V_{q}$ is proportional to $W_{d}$, and the slope gives $D$; this property is exploited later, in order to calibrate the measures.

In the second example, the finger is in contact with the vibrating plate; due to the acoustical impedance of the finger pulp, the contact force depends on the vibration itself. If we consider a typical model that includes the internal damping of the skin (denoted by $\left.D_{f}\right)$ and the vibrating mass, $\left(M_{f}\right)$
[5], the acoustical finger force is sinusoidal, and writes $f_{r}=$ $D_{f} \dot{w}+M_{f} \ddot{w}$, leading to:

$$
F_{r d}=-M_{f} \omega^{2} W_{d} \quad, \quad F_{r q}=D_{f} \omega W_{d}
$$

The acoustical force is then shared between the axis: $d$ axis contains the inertial force while $q$ axis contains the damping force.

In the last example, we consider intermittent contact. When the contact is intermittent, $f_{r}$ shows pulses at the frequency of the vibration, and with a phase shift named $\Phi$ [3]. Each time the skin bounces on the plate, the force $f_{r}$ increases before returning to 0 when the skin is detaching from the plate. For illustration, we consider perfect rebound of the skin: the contact time equals zero, and the contact force is infinite when the plate touches the finger. Therefore, we write $f_{r}$ as a Dirac comb distribution $\Delta($.$) , with f_{r}=$ $f_{0} \sum_{n=-\infty}^{\infty} \delta\left(t-n \frac{2 \pi}{\omega}-\Phi\right)$.
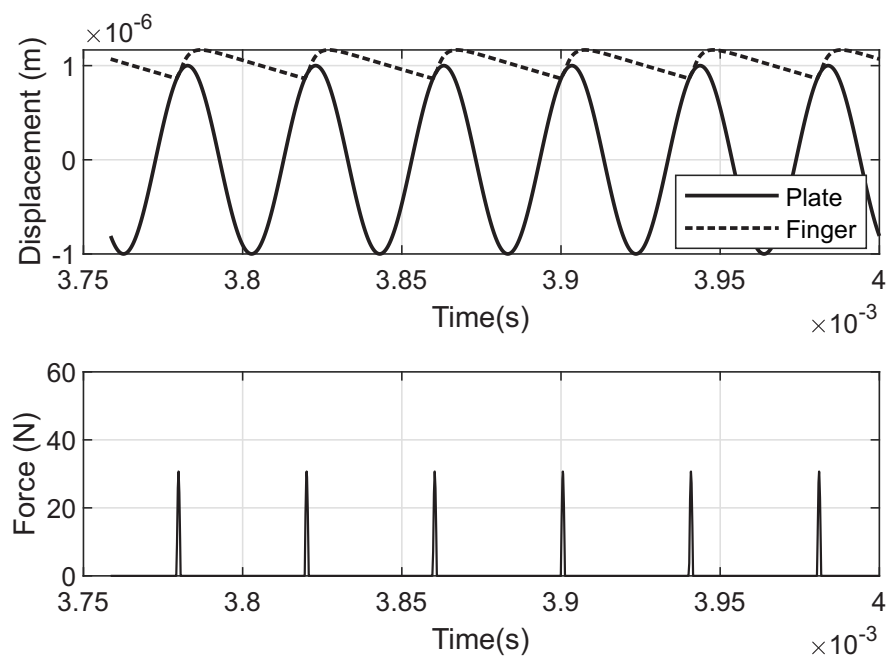

Fig. 2. Evolution of $f_{r}$ in case of an ideal intermittent contact.

The acoustical finger force $f_{r}$ contains a fundamental, which is at the resonance pulsation of the plate, and harmonics. Due to the resonant behaviour of the plate, the harmonic doesn't influence the plate's vibration, and therefore, only the fundamental can be observed with the equation 7 . In the particular case of ideal intermittent contact condition, the fundamental of $f_{r}$ writes:

$$
\tilde{F}_{r d}=2 f_{0} \cos (\Phi) \quad, \quad \tilde{F}_{r q}=2 f_{0} \sin (\Phi)
$$

Hence, when the contact is intermittent, the observation of the acoustical finger force is not related to the acoustical impedance of the finger pulpe, and the components along the axis $d$ and $q$ are not related to a damping or a mass. However, the phase shift, which can be calculated from $\operatorname{atan}\left(\frac{\tilde{F}_{r q}}{\tilde{F}_{r d}}\right)$ is related to the time at which the contact occurs.

\section{EXPERIMENTAL SETUP AND PROTOCOL}

\section{A. Material}

The setup is as shown in figure 3 . An aluminum beam $\left(18 \times 119 \times 2 \mathrm{~mm}^{3}\right)$ is covered with a hydrophobic surface, 

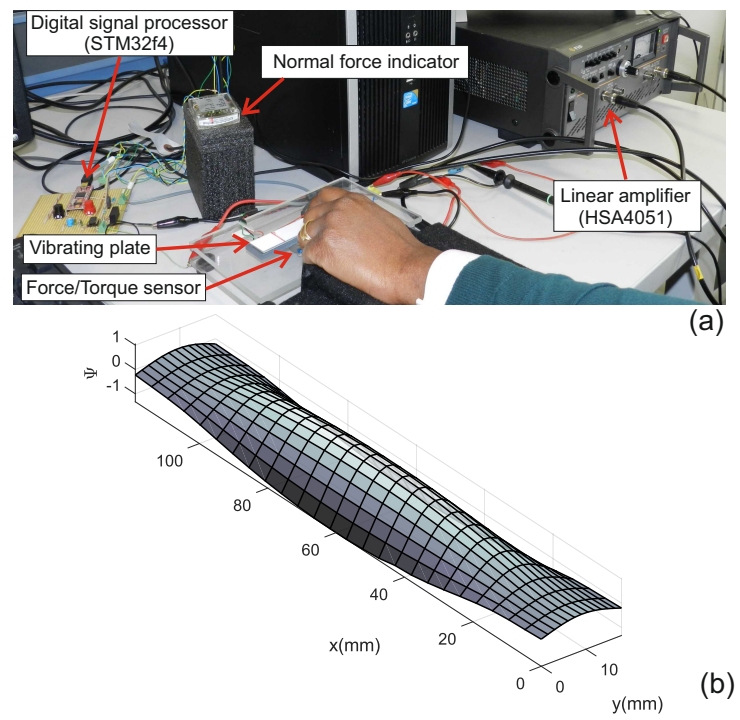

(a)

Fig. 3. The experimental setup; (a) is the vibrating plate and (b) is its deformation mode shape $\Phi(x, y)$.

and sustained by a structure equipped with a 6-dimensional force sensor (ATI Nano 43); an analog Standard Volume Indicator (SVI) gives an indication of the pressing force, with a specific guideline at $0.4 \mathrm{~N}$. Four piezoelectric ceramics of magnitude $16 \times 4 \times 0.5 \mathrm{~mm}^{3}$ are glued to the opposite surface of the beam to produce the vibration. The geometry of the beam, magnitude and position of the piezoelectric sensors are designed and simulated with an FE analysis in order to attain a pre-defined normal wave vibration mode at the plate's resonance frequency. We exploit the first bending mode of the beam, which is obtained with a resonant frequency of $24870 \mathrm{kHz}$. Closed loop control of the vibration amplitude of the beam is achieved thanks to the use of a Digital Signal Processor (STM32F4 from ST Microelectronics). The acoustical force are observed online, and sent to a main computer through a serial connection for data collection. The vibration control is achieved at $10 \mathrm{kHz}$. An external power amplifier (HSA 4051 from NF, Japan) amplifies the controller's output up to $200 \mathrm{~V}$ peak-peak.

The parameter $N$ of the plate has been identified using the methodology described in [11] and its value is $N=$ $0.02 N / V$. The no-load test measurement presented figure 4 has been used to identify $D=0.2848 N$.s. $\mathrm{m}^{-1}$.

\section{B. Experimental procedure}

Twelve healthy participants (3 females) aged from 22 to 60 participated to the experiments. They were asked to thoroughly wash and dry their hand before the tests. All participants gave written informed consent. The investigation conformed to the principles of the Declaration of Helsinki and experiments were performed in accordance with relevant guidelines and regulations.

Prior to any experiment, the participants train themselves to apply a constant pressure of $0.4 \mathrm{~N}$ on the plate, by following the indication of the SVI.

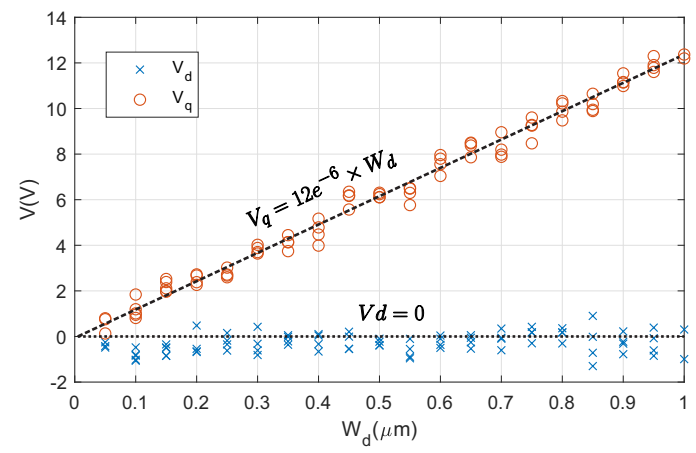

Fig. 4. No-load relation between the applied voltage and the reference $W_{d}$

Two experiments were designed in this work. In the first experiment, the participants placed their finger in the middle of the plate, standstill, with an angle of 45deg, yet pushing with $0.4 \mathrm{~N}$ with their finger on the plate. The location for the measurement has been chosen to have an almost constant deformation mode shape and to maximize the relation 2 . During the experiment, the vibration amplitude $W_{d}$ varies from $50 \mathrm{~nm}$ to $1000 \mathrm{~nm}$ peak in 20 steps of $50 \mathrm{~nm}$ every $100 \mathrm{~ms}$, back and forth; 5 cycles are achieved, and the observed values of $F_{r d}$ and $F_{r q}$ are recorded at the end of each step.

In the second experiment, the participant moved their finger from left to right, in a lateral movement. They were asked to keep an inclination of $45 \mathrm{deg}$ and a pressing force of $0.4 \mathrm{~N}$. To control the finger's speed, a metronome emits sound at $1 \mathrm{~Hz}$ and participants are asked to move their finger between two lines marked on the plate in two metronome beeps. The nominal speed is then equal to $70 \mathrm{~mm} / \mathrm{s}$. During this try, $10 \mathrm{~Hz}$ vibration amplitude modulation from 0 to 1000 $\mathrm{nm}$ peak are produced on the plate, and the normal and tangential forces are recorded with a sampling rate of $100 \mathrm{~Hz}$.

The second experiment is achieved right after the second one, to keep the same experimental conditions, to get benefit of the training of the finger inclination and pressing force, as well as to keep the participants' skins in the same condition,

\section{RESUlTS}

\section{A. Result from experiment 1}

During the tests, the participants controlled the pressing force with a higher pressure than required (mean $=0.515 \mathrm{~N})$, but maintained it almost constant over the test $\left(\mathrm{SD}_{i} 0.07 \mathrm{~N}\right.$ over all participants). The result of the first experiment shows common features and differences from one user to the other, and the figure 5 presents the estimated values for all participants.

We notice that the observed acoustical finger force does not necessarily equally share on axis $d$ and $q$, and that for some participants, there is a saturation in axis $d$ which do not appear on the axis $q$. In order to establish a comparison between each participant, we have reported in figure 6 their maximal acoustical finger force i.e. $\max \left(\sqrt{\tilde{F}_{r d}^{2}+\tilde{F}_{r q}^{2}}\right)$. 

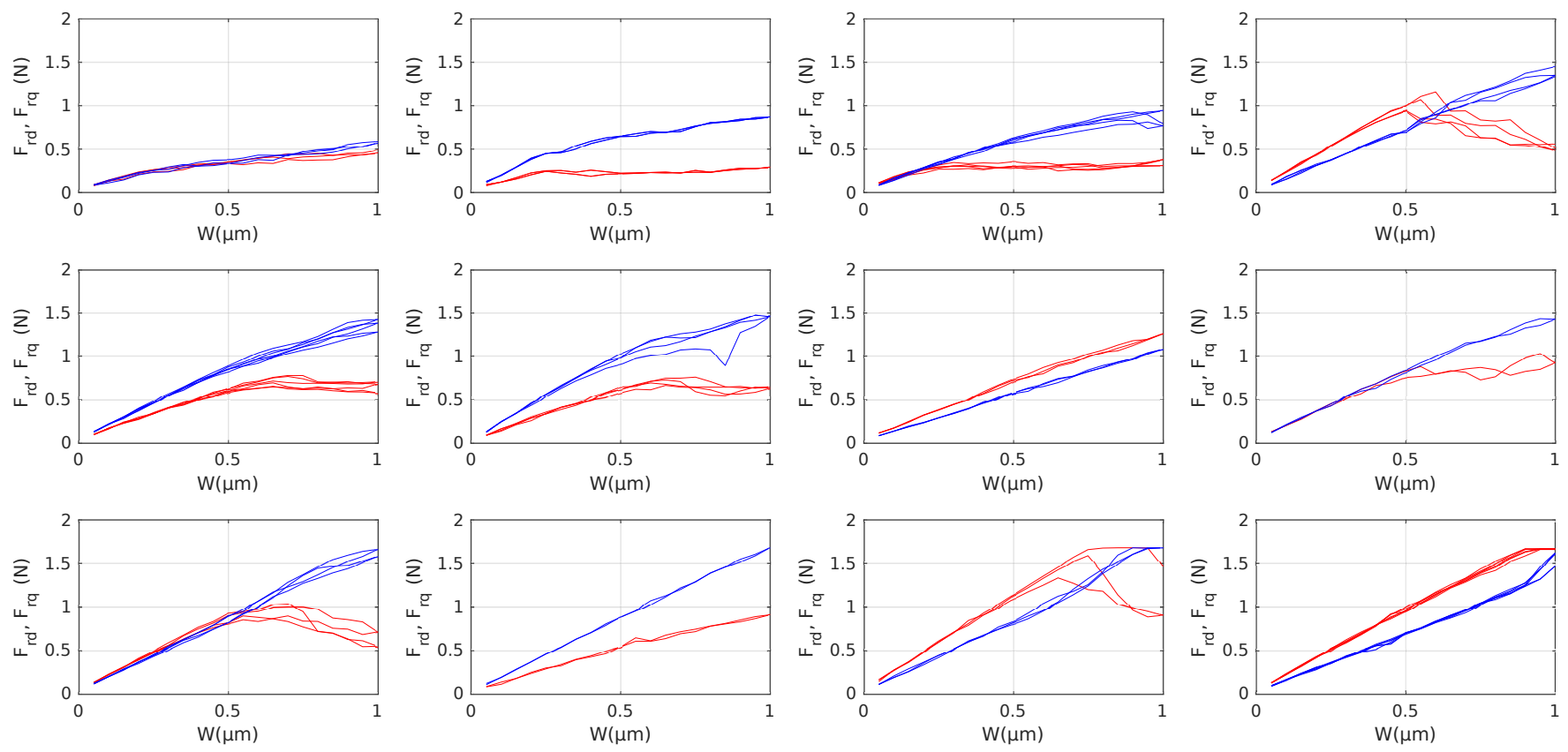

Fig. 5. Estimated values of $\tilde{F}_{r d}$ (red) and $\tilde{F}_{r q}$ (blue) as a function of $W$ for every participants;

The results are sorted in the same order as for the figure 5 .

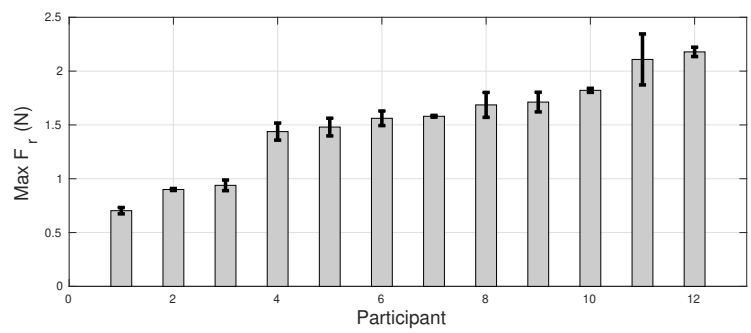

Fig. 6. Maximal acoustical finger force observed for each participant.

The value of the maximal acoustical force depend on each participant, and this figure is relevant to discriminate them. Moreover, the measure is robust, the standard deviation is small compared to the mean value over the five cycles of measurement.

\section{B. Result from experiment 2}

The results of the second experiment helped to characterize the friction modulation for each participant. The figure 7 depicts a typical friction measurement when the participant is sliding the finger over the tactile plate.

A positive friction coefficient (denoted as $\mu$ ) is obtained when the finger is sliding from left to right, while a negative $\mu$ is obtained in the reverse direction. The modulation of $\mu$ is due to to the modulation of the vibrating amplitude $W$ while sliding.

To characterize the friction modulation, we calculate, from the records of each participant, the maximum of $\mu$, named $\mu_{\max }$ and the friction contrast denoted $\Delta \mu$. Since the value may change from one period of friction modulation to an other, we average the measurement over 6 periods

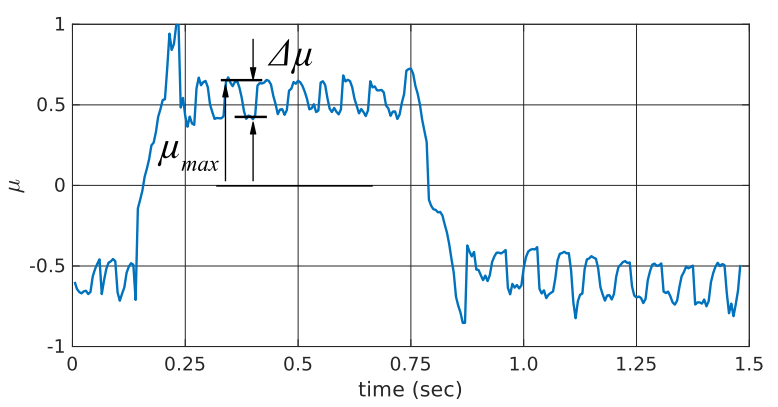

Fig. 7. Typical friction coefficient $\mu$ as a function of time for one participant.

of friction modulation, either for the positive or negative coefficients. The resulting values are given in the figure 8 for all participants, sorted in the same order as for the figure 5 .

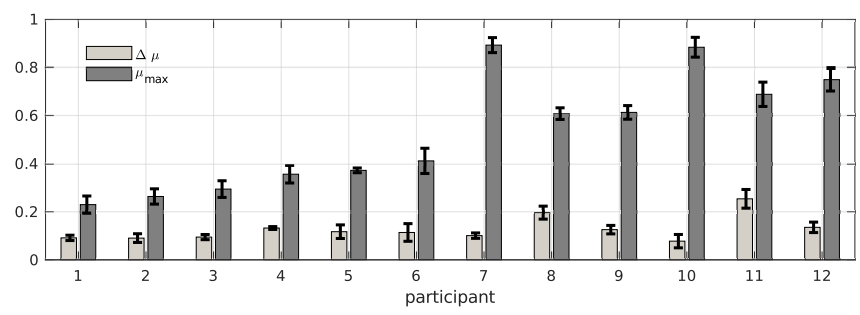

Fig. 8. Maximum of friction $\mu_{\max }$ and friction contrast $\Delta \mu$ for each participant.

This figure shows two groups of participants: The first group of participants (from 1 to 6) have a low friction coefficient (below 0.5) without vibrations; this group also have a low value of maximal acoustical finger force (less than 1.6). The other group of participants (from 7 to 12) 
have a high level of friction (above 0.5) and a maximum force of $1.5 \mathrm{~N}$. Therefore, it seems reasonable to consider a correlation between the acoustical force measurement, and the level of friction with and without ultrasonic vibration.

\section{Correlation between acoustical finger force measurement and friction reduction}

We report in figure 9 the friction contrast $\Delta \mu$ as a function of the maximal acoustical finger force, and for each participant.

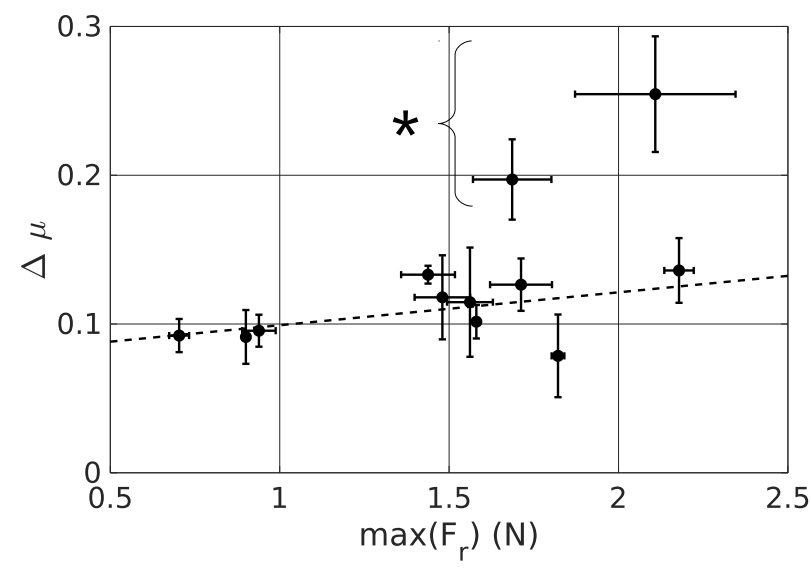

Fig. 9. $\Delta \mu$ as a function of $\max \left(F_{r}\right)$ for each participant, and a linear fitting for the data; the participants marked by $*$ have been removed from the model.

We can propose a linear model to the data (Pearson correlation is 0.541 ), that helps to predict the friction reduction as a function of the maximal acoustical finger force. A linear regression gives $\Delta \mu=A_{\Delta} \times \max \left(F_{r}\right)+B_{\Delta}$, with $A_{\delta}=0.022$ and $B_{\delta}=0.071$ (norm of residual: 0.0506). To obtain this model, we have excluded two participants ( 8 and 11); including them would have resulted in other coefficients, but higher norm of residuals. The proposed model suits well for the 10 participants chosen for the linear regression.

We also depicts $\mu_{\max }$ as a function of $\max \left(F_{r}\right)$ in the figure 10. Also, we present a linear fitting to the data.

Here also, two participants have been removed from the linear fitting (7 and 10); The Person Correlation is 0.941 , so a linear regression that gives $\mu_{\max }=A_{\mu} \times \max \left(F_{r}\right)+B_{\mu}$, with $A_{\mu}=0.358$ and $B_{\mu}=-0.067$ (norm of residuals: 0.192 ) is proposed. This model can predict the coefficient of friction without ultrasonic vibration for the 10 participants used for the linear regression, with a maximal error of 0.09 .

\section{DISCUSSION}

The measurement of the fundamental acoustical finger force has been achieved on a light Digital Signal Processor, and does not require off line calculation. We obtained measures that have in common that the acoustical finger force increases as the vibration amplitude increases. However, we remark in figure 5 that for some participants, the force along the axis $d$ saturates. We believe that this change in force slope corresponds to the beginning of the intermittent contact. The

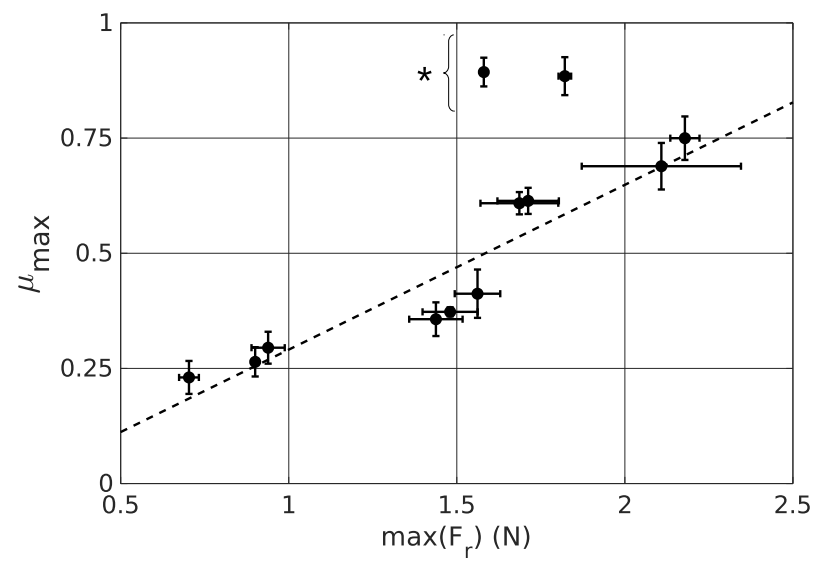

Fig. 10. $\mu_{\max }$ as a function of $\max \left(F_{r}\right)$ for each participant, and a linear fitting for the data; the participants marked by * have been removed from the model.

amount of vibration amplitude from which the skin starts to bounce is different from one participant to the other. This might be due to the different mechanical skin parameters. Indeed, it has been shown that intermittent contact occurs for higher vibration amplitude for soft skin [3]. Surprisingly, the level of force is much higher than the pressing force (around $0.4 \mathrm{~N}$ ). The fact that intermittent contact produces pulses of forces, introducing a factor 2 into the fundamental acoustical finger force as shown in the equation 8 is not enough to explain the very large values obtained. The value of $N$ might be overestimated, and further investigation are needed to address this issue.

The figure 8 shows that the participant are shared into two categories. Those with a high friction $\mu_{\max }$ have high acoustical finger forces, and vice versa. This correlation has been observed on the curve of the figure 10. This can be explained by the moisture level of the participants' finger. Indeed, [12] demonstrate that higher level of moisture leads to higher level of friction coefficient, for all surfaces (without ultrasonic vibrations), while in the same time, [13] shows that a moist skin also have lower stiffness than a dry one, and thus needs higher vibration amplitude to obtain the intermittent contact. Two participants (7 and 10) however have higher coefficient of friction than what is expected from the proposed model. The behaviour of $\tilde{F}_{r}$ is different: it does not saturate, as if the finger skin has not reached the intermittent contact.

Finally, the measurements have shown that the friction contrast can be correlated to the acoustical finger force, for the surface considered in this paper.

\section{CONCLUSIONS}

A conclusion section is not required. Although a conclusion may review the main points of the paper, do not replicate the abstract as the conclusion. A conclusion might elaborate on the importance of the work or suggest applications and extensions. 


\section{APPENDIX}

Appendixes should appear before the acknowledgment.

\section{ACKNOWLEDGMENT}

\section{Thanks IRCICA And Maxime}

\section{REFERENCES}

[1] L. Winfield, J. Glassmire, J. E. Colgate, and M. Peshkin, "T-pad: Tactile pattern display through variable friction reduction," in World Haptics Conference. IEEE, 2007, pp. 421-426.

[2] M. Biet, F. Giraud, and B. Lemaire-Semail, "Squeeze film effect for the design of an ultrasonic tactile plate," IEEE transactions on ultrasonics, ferroelectrics, and frequency control, vol. 54, no. 12, pp. 2678-2688,2007.

[3] E. Vezzoli, Z. Vidrih, V. Giamundo, B. Lemaire-Semail, F. Giraud, T. Rodic, D. Peric, M. Adams "Friction reduction through ultrasonic vibration part 1: Modelling intermittent contact", in IEEE transactions on haptics, vol 10(2), 2017, pp. 196-207.

[4] M. Wiertlewski, R. Fenton Friesen and J.E. Colgate, "Partial squeeze film levitation modulates fingertip friction", in Proceedings of the National Academy of Sciences, 2016, vol 113(33) 9210-9215.

[5] R. Fenton Friesen, M. Wiertlewski and J.E. Colgate, "The role of damping in ultrasonic friction reduction" in IEEE Haptics Symposium (HAPTICS). IEEE, 2016, pp. 167-172.

[6] W. Ben Messaoud, M.-A. Bueno, B. Lemaire-Semail, "Relation between human perceived friction and finger friction characteristics", in Tribology International vol 98, 2016, pp 261-269.

[7] Y.M. Khurram Saleem, C. Yilmaz, C. Basdogan and Y. tarihi "Psychophysical Evaluation of Change in Friction on an UltrasonicallyActuated Touchscreen", in IEEE Transactions on Haptics vol 11(4), 2018, pp 599-610.

[8] J. Monnoyer, E. Diaz, C. Bourdin and M. Wiertlewski, "Perception of ultrasonic switches involves large discontinuity of the mechanical impedance", in IEEE transactions on haptics vol 11(4), 2018, pp 579589.
[9] F. Giraud, M. Amberg and B. Lemaire-Semail, "Design and control of a haptic knob", in Sensors and Actuators A: Physical, vol 196, elsevier, 2013, pp 78-85.

[10] S. Ghenna, F. Giraud, C. Giraud-Audine and M. Amberg, "Vector Control Of Piezoelectric Transducers and Ultrasonic Actuators" in IEEE Transactions on Industrial Electronic vol 65(6), 2017, pp 4880 - 4888.

[11] S. Ghenna, F. Giraud, C. Giraud-Audine, M. Amberg and B. LemaireSemail, "Modelling, identification and control of a Langevin transducer", in IEEE International Workshop in Electronics, Control, Measurement, Signals and their Application to Mechatronics (ECMSM), 2015, pp 1-6.

[12] D. Gueorguiev, E. Vezzoli, A. Mouraux, B. Lemaire-Semail, and J.-L. Thonnard, "the tactile perception of transient changes in friction", in Journal of The Royal Society Interface vol 14(137), 2017, pp 1-10.

[13] J. v. Kuilenburg, M. A. Masen, and E. v. d. Heide, "Contact modelling of human skin: What value to use for the modulus of elasticity?", in Proceedings of the Institution of Mechanical Engineers, PartJ: Journal of Engineering Tribology, vol. 227(4), 2012, pp. 349-361 